\title{
Foxp $3^{+}$cells are running the show in patients with surgically resected nonsmall cell lung cancer
}

\author{
Nathalie Chaput ${ }^{1,2,4}$ and Aurelien Marabelle $1,3,5$
}

Affiliations: 'Gustave Roussy Cancer Campus, Villejuif, France. ${ }^{2}$ Laboratory of Immune monitoring in Oncology UMS 3655 CNRS/US 23 INSERM Gustave Roussy Cancer Campus, Villejuif, France. ${ }^{3}$ Center of Clinical Investigations in Biotherapies of Cancer (CICBT) 1428, Villejuif, France. ${ }^{4}$ Cell Therapy Unit, Gustave Roussy Cancer Campus, Villejuif, France. ${ }^{5}$ Drug Development Department, Gustave Roussy Cancer Campus, Villejuif, France.

Correspondence: Nathalie Chaput, Laboratoire d'Immunomonitoring en Oncologie, UMS 3655 CNRS / US 23 INSERM, Laboratoire de Thérapie Cellulaire, Gustave Roussy Cancer Campus, 114 rue Edouard Vaillant, 94805 Villejuif, France. E-mail: nathalie.chaputagustaveroussy.fr

(y) @ERSpublications

Foxp3 microlocalisation dictates prognosis in NSCLC; $\mathrm{CD8}^{+} \mathrm{T}$-cells may influence prognosis in Treg-infiltrated tumours http://ow.ly/T9PwJ

Tumor lymphocyte infiltrates (TILs) have a significant impact on multiple cancers prognosis, but the diversity of TILs and the methodological challenges to analyse TIL infiltrates in a reliable manner render difficult the interpretation of data explaining that to date TIL infiltration is not used in daily clinical practice.

O'Callaghan et al. [1] analysed the micro-localisation of T-cells (CD3 and CD8 positive cells) and regulatory T-cells (Treg; Foxp3-positive cells) in tumour specimens from 196 patients with stage IA-IIIA surgically resected nonsmall cell lung cancer (NSCLC). The authors selected patients who did not receive adjuvant treatment; this is of particular relevance since chemotherapy can modify immune functions [2] and could act as a confounder with respect to outcome. They determined the absolute number of immunohistochemistry-stained lymphocytes on whole-slide tumour specimens using a novel cell detection algorithm and reported as numbers of cells per $\mathrm{mm}^{2}$ of tumour and stroma region. They calculated for each marker (CD3; CD8 and Foxp3) the ratio of corresponding tumour islet and stroma (TI/S) counts. Patients could then be classified as $\mathrm{CD}^{+\mathrm{HIGH}}$ or $\mathrm{CD}^{+\mathrm{LOW}}, \mathrm{CD}^{+\mathrm{HIGH}}$ and $\mathrm{CD} 8^{+\mathrm{LOW}}$ and Foxp3 $3^{+\mathrm{HIGH}}$ and Foxp $3^{+ \text {LOW }}$ depending on whether TI/S ratio was above or below median value. Thus this study takes into account the regional distribution of lymphocyte subsets. Indeed, ratio allows for more specific information considering the infiltrate as a whole including the ability of lymphocytes to enter the tumour. The study of prognostic relevance of lymphocyte infiltration showed that the regional distribution of lymphocyte subsets may help identifying patients at risk of early relapse in a large population of surgically resected NSCLC. A positive association between $\mathrm{CD} 8^{+\mathrm{HIGH}}$ and survival was observed with 59\% of patients alive after 5 years versus $34 \%$ for those patients with a CD8 ${ }^{+ \text {LOW }}$ pattern $(\mathrm{p}<0.001)$. Foxp $3^{+\mathrm{HIGH}}$ was associated with a worse outcome with $20 \%$ of patients alive after 5 years versus $69 \%$ for those patients with a Foxp $3^{+\mathrm{LOW}}$ pattern $(\mathrm{p}<0.001)$. Finally, no significant link with prognosis was observed with CD3 TI/S ratio. This is probably explained by the fact that CD3 allows identification of all $\mathrm{T}$ subsets, therefore a mixture of effector and Treg. Very interestingly, O'CALLAGHAN et al. [1] have also analysed the relationship between the absolute lymphocytes counts (cells per $\mathrm{mm}^{2}$ ) and prognosis. As would be expected, a high number of $\mathrm{CD}^{+}$cells and low number of Foxp $3^{+}$cells in the tumour islets were associated with a significant survival advantage while high number of Foxp $3^{+}$cells was associated with a worse outcome. Less expected was that high numbers of $\mathrm{CD}^{+}$and $\mathrm{CD} 8^{+}$cells in the stroma were associated with a poor outcome and high number of $\mathrm{CD}^{+}$cells in tumour islets had no significant

Received: Sept 252015 | Accepted after revision: Sept 252015

Conflict of interest: None declared

Copyright @ERS 2015 
relationship with prognosis. Altogether these data demonstrate that taking into account the balance between peri- and intra-tumoural lymphocytes is critical for predicting survival in surgically resected NSCLC. CD8 and Foxp3 TI/S ratios were both independent predictors of survival and were stronger prognostic value than tumour stages according to the International System of Staging for Lung Cancer [3].

The prognostic value of CD8 and Foxp3 TI/S ratios was also explored. The four possible combinations $\left(\mathrm{CD}^{+\mathrm{HIGH}} / \mathrm{Foxp}^{+\mathrm{HIGH}}, \mathrm{CD}^{+\mathrm{HIGH}} / \mathrm{Foxp}^{+\mathrm{LOW}}, \mathrm{CD}^{+\mathrm{LOW}} / \mathrm{Foxp}^{+\mathrm{HIGH}}\right.$ and $\left.\mathrm{CD}^{+\mathrm{LOW}} / \mathrm{Foxp}^{+\mathrm{LOW}}\right)$ were examined with regard to prognosis. Surprisingly, tumour specimens with a low Foxp3 TI/S ratio have a favorable prognosis regardless of the CD8 TI/S ratio. Indeed the 5 -year survival of $\mathrm{CD} 8^{+\mathrm{HIGH}} / \mathrm{Foxp}^{+\mathrm{LOW}}$ and $\mathrm{CD} 8^{+\mathrm{LOW}} / \mathrm{Foxp}^{+\mathrm{LOW}}$ was 73 and $77 \%$ respectively and median survival was not reached in these two groups. By contrast, tumour specimens with a high Foxp3 TI/S ratio have a worse prognosis but influenced by the CD8 TI/S ratio. Indeed, the median survival in the group of patients with the $\mathrm{CD}^{+\mathrm{HIGH}} / \mathrm{Foxp}^{+\mathrm{HIGH}}$ was 31 months versus 17.2 months in the group of patients with $\mathrm{CD} 8^{+\mathrm{LOW}} /$ Foxp ${ }^{+\mathrm{HIGH}}$. This indicates that Foxp3 micro-localisation dictates the prognosis and that CD8 T-cells may influence the prognosis only in tumours where the Treg TI/S ratio is high (figure 1). This work is in accordance with other studies showing the impact of tumour infiltrating Treg cells on patient with surgically resected NSCLC. Hanagiri and colleagues screened 131 patients who underwent complete surgical resection for stage I NSCLC [4]. The clinical significance of the relative expression of Foxp3 in the

a)

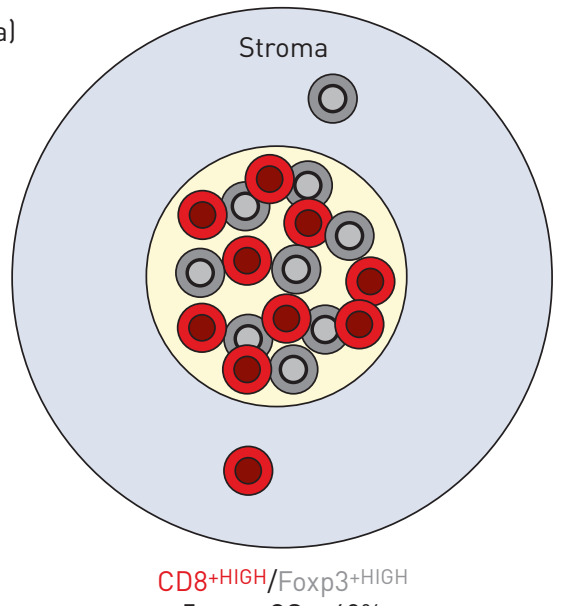

5 -year OS $\approx 40 \%$

b)

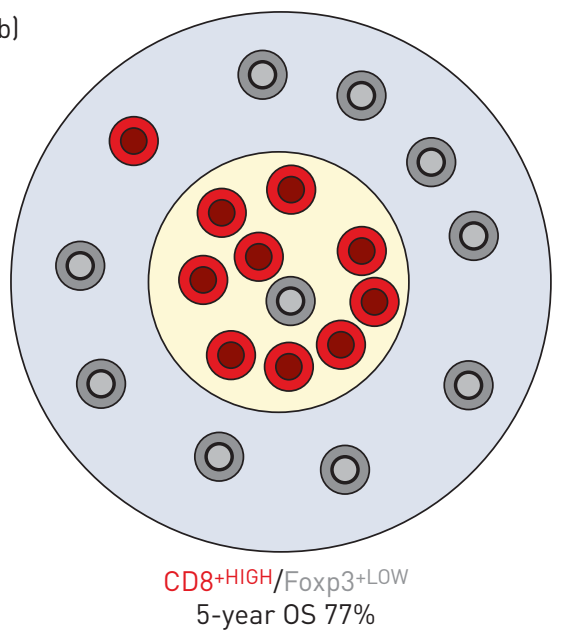

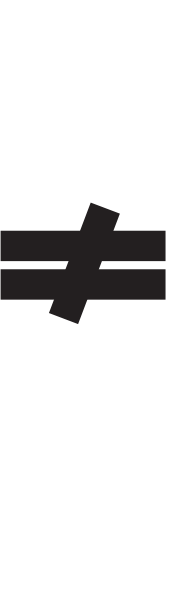
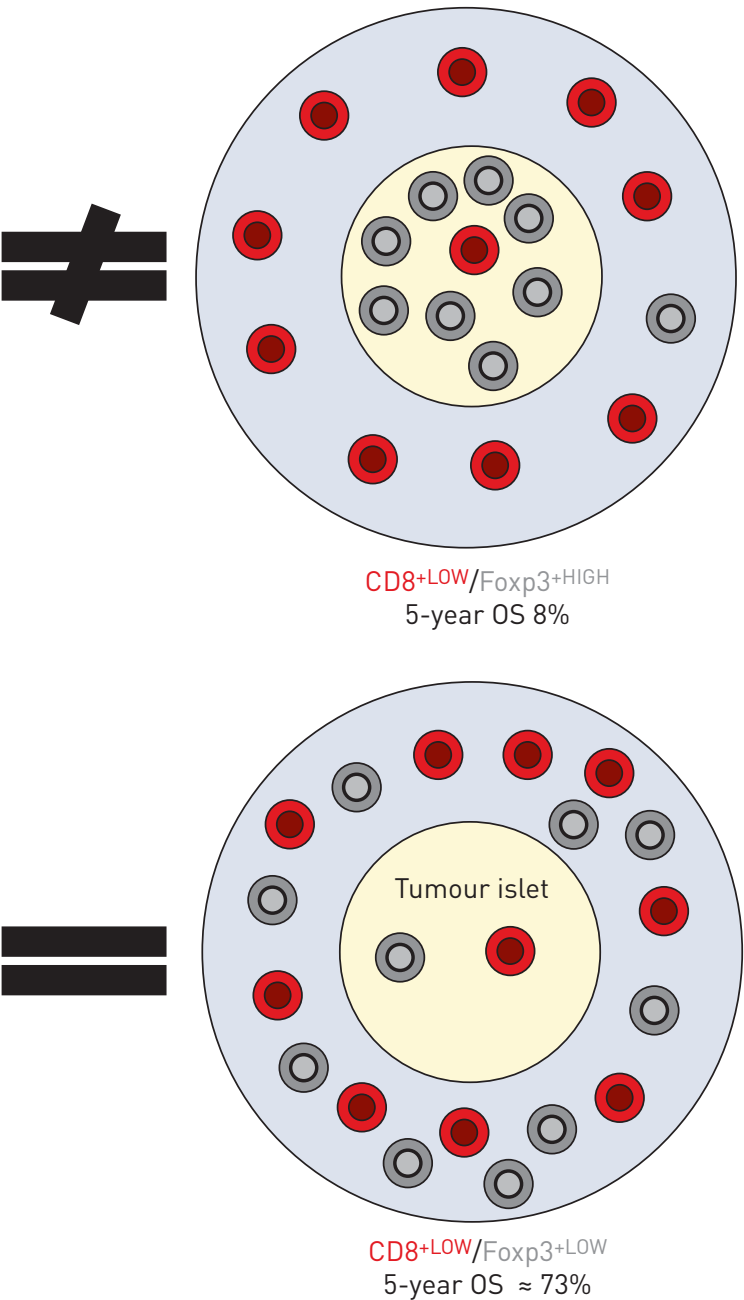

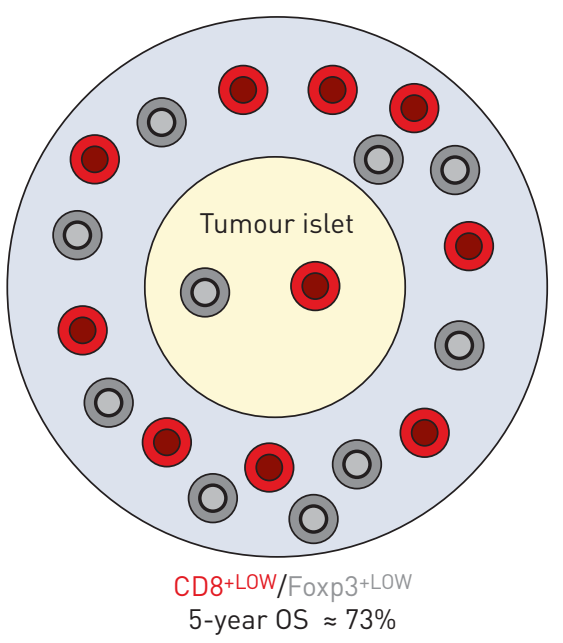

FIGURE 1 Schematic representation of the prognostic value by combining CD8 and Foxp3 tumour islet and stroma (TI/S) ratios. a) Foxp3 TI/S ratio is high in both cases (left and right panels) but the CD8 TI/S ratio is high (left panel; CD8 ${ }^{+H I G H} / F o x p 3^{+H I G H}$ ) or low (right panel; CD8 $8^{+L O W} / F o x p 3^{+H I G H}$ ). Both patterns have a different 5 -year survival. CD8 TI/S ratio highly influences patient outcomes with a 31-months median survival when CD8 TI/S ratio is high and only 17 months median survival when the CD8 TI/S ratio is low. b) Foxp3 TI/S ratio is low in both cases (left and right panels) but the CD8 TI/S ratio is high (left panel; $\mathrm{CD}^{+\mathrm{HIGH}} / \mathrm{Foxp}^{+\mathrm{LOW}}$ ) or low (right panel; CD8 $8^{+\mathrm{LOW}} / \mathrm{Foxp}^{+\mathrm{LOW}}$ ). Both patterns have a quiet similar 5 -year survival. CD8 TI/S ratio does not significantly influence patient outcomes (77 and 73\% 5-year survival respectively). 
regional lymph nodes was explored. High expression of Foxp3 in the regional lymph nodes was associated with a poor prognostic factor in these patients. Previous study using flow cytometry demonstrated that the frequency of Foxp $3^{+}$cells in the regional lymph nodes was a significance prognostic factor in patients who underwent surgical resection for NSCLC [5]. Another recent work has shown that peripheral blood Foxp $3^{+}$cells could serve as a prognostic biomarker in NSCLC patients [6]. These works bring the first rationale for immune biomarkers in patients with surgically resected NSCLC that could help determining which individuals are at a higher risk of relapse after surgical resection, and who are indicated for treatment and follow-up examinations. Also Foxp3 pattern of expression may reflect the tumour immunogenicity and correlate with the mutational load of NSCLC tumours [7]. Such observation could point out that immunogenic tumours may have a poorer outcome probably due to an active participation of immunosuppressive mechanisms participating in the tumour outgrowth, survival, proliferation and dissemination. These studies support the hypothesis that Treg play a pivotal role in NSCLC, and that CD8 ${ }^{+}$ T-cells are critical but their action is limited by the presence of Foxp $3^{+}$cells in these NSCLC patients. The characterisation of these differentially located tumour-infiltrative T-cells would be of great interest to know where the subsets of tumour-specific T-cells preferentially reside in the tumour bed. This is of peculiar interest in the current development of PD-1 and CTLA-4 immune checkpoint-targeted antibodies in NSCLC. Indeed, CTLA-4 is highly expressed at the membrane of tumour-specific Treg [8, 9], and PD-1 is a marker of exhausted tumour-specific CD8+ T-cells [10] and is also expressed by tumour-infiltrative Treg. Therefore, future studies of immune contexture in NSCLC should at least include an analysis of PD-1 expression on $\mathrm{CD}^{+}$and Foxp3 $3^{+}$T-cells. Such analysis could be performed on tumour biopsies prior a neo-adjuvant anti-PD-1/PD-L1 monotherapy. This would allow performing subsequent correlative studies between immune infiltrative patterns, response rates and level of immune reactivation (in the surgical piece). Indeed, it would make sense that anti-PD-1 monotherapy or the combination of anti-PD-1 and anti-CTLA-4 antibodies would provide different response rates within the four subsets of tumour types described by O'Callaghan et al. [1] (figure 1).

Knowing the multiple ongoing and upcoming large immunotherapy trials in lung cancers, there are good chances that the groundwork for immune therapeutic stratification of NSCLC patients will emerge over the next couple of years.

\section{References}

1 O'Callaghan R. Tumour islet Foxp3 ${ }^{+}$T-cell infiltration predicts poor outcome in nonsmall cell lung cancer. Eur Respir J 2015; 46: 1762-1772.

2 Shinto $\mathrm{E}$, Hase $\mathrm{K}$, Hashiguchi $\mathrm{Y}$, et al. CD8+ and FOXP3+ tumor-infiltrating $\mathrm{T}$ cells before and after chemoradiotherapy for rectal cancer. Ann Surg Oncol 2014; 21: Suppl. 3, S414-S421.

3 Mountain CF. Revisions in the International System for Staging Lung Cancer. Chest 1997; 111: 1710-1717.

4 Hanagiri T, Fukumoto M, Koyanagi Y, et al. Regulatory T-cells and micrometastasis in lymph nodes of stage I NSCLC. Anticancer Res 2014; 34: 7185-7190.

5 Hanagiri T, Shigematsu Y, Shinohara S, et al. Clinical significance of the frequency of regulatory $\mathrm{T}$ cells in regional lymph node lymphocytes as a prognostic factor for non-small-cell lung cancer. Lung Cancer 2013; 81: 475-479.

6 Hasegawa T, Suzuki H, Yamaura T, et al. Prognostic value of peripheral and local forkhead box P3 regulatory T cells in patients with non-small-cell lung cancer. Mol Clin Oncol 2014; 2: 685-694.

7 Rizvi NA, Hellmann MD, Snyder A, et al. Cancer immunology. Mutational landscape determines sensitivity to PD-1 blockade in non-small cell lung cancer. Science 2015; 348: 124-128.

8 Marabelle A, Kohrt H, Sagiv-Barfi I, et al. Depleting tumor-specific Tregs at a single site eradicates disseminated tumors. J Clin Investig 2013; 123: 2447-2463.

9 Simpson TR, Li F, Montalvo-Ortiz W, et al. Fc-dependent depletion of tumor-infiltrating regulatory $\mathrm{T}$ cells co-defines the efficacy of anti-CTLA-4 therapy against melanoma. J Exp Med 2013; 210: 1695-1710.

10 Tumeh PC, Harview CL, Yearley JH, et al. PD-1 blockade induces responses by inhibiting adaptive immune resistance. Nature 2014; 515: 568-571. 